\title{
Quantifying the global burden of ischaemic heart disease
}

In most parts of the world, age-standardized morbidity and mortality from ischaemic heart disease (IHD) decreased between 1980 and 2010. However, population growth and ageing mean that the absolute burden of IHD increased over this 30 -year period. These data from the Global Burden of Disease (GBD) 2010 Study emphasize the continued need to implement effective primary and secondary prevention measures for cardiovascular disease, particularly in low-income and middle-income countries.

The GBD 2010 Study is an ongoing, international collaboration between nearly 500 researchers from more than 300 institutions worldwide. Two papers have now been published in Circulation to quantify the global burden of IHD. The lead author, Andrew Moran, is particularly interested in "the effects of dynamic social changes on health-changes brought on by migration, acculturation, urbanization, and economic development and stresses".

Using widescale, systematic reviews of published literature and complex meta-regression modelling techniques, Moran and colleagues sought to quantify mortality from IHD, and morbidity associated with three major IHD sequelae: nonfatal acute myocardial infarction (AMI), angina, and ischaemic heart failure. In most parts of the world, agestandardized IHD mortality has decreased since 1980, particularly in high-income regions, such as Australasia, Western Europe, and North America, which is testament to effective prevention and treatment strategies. However, some regions, such as Eastern Europe, Central Asia, and East Asia, have experienced an increase in age-standardized IHD mortality, highlighting the need for the effective control of established cardiovascular risk factors in these areas. Furthermore, demographic trends in population growth and ageing mean that the absolute number of deaths from IHD is steadily increasing, from 4.5 million in 1980 to $>7.0$ million in 2010 .

The global burden of IHD comprises both years of life lost as a consequence of premature death, but also impaired quality of life caused by IHD sequelae. The global, age-standardized incidence of nonfatal AMI decreased between 1990 and 2010, particularly in Australasia, Western and Central Europe, and North America. However, the incidence of nonfatal AMI increased in some regions, such as Eastern Europe. The age-standardized prevalence of angina also decreased globally, whereas the global burden of ischaemic heart failure increased slightly in men and remained constant in women. Overall, the absolute global burden of IHD is estimated to have increased by 29 million disability-adjusted life-years between 1990 and 2010.

Epidemiological research, such as the GBD 2010 Study, is inevitably hampered by the quality and quantity of data available, particularly from low-income and middle-income countries, as well as necessary assumptions in the modelling algorithms used. "IHD measurement problems," say the investigators, "would

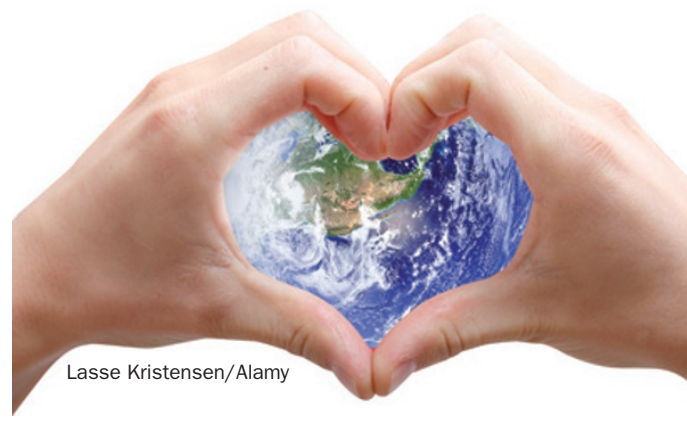

best be addressed by improved methods of health surveillance, including investment in better interview or objective diagnostic screening methods applicable to low-income settings." Indeed, in an editorial that accompanied the study reports, Salim Yusuf and Martin McKee point out that "countries that have good documentation of their mortality and morbidity are also the ones that develop policies and care systems that improve health in their countries". According to Dr Moran, the GBD researchers are continuing to work on estimates for 2013 to "fulfill [their] mission of improving the world's health and health equality".

Gregory B. Lim 